\title{
3 La sezione conclusiva del secondo libro
}

Multaque post mundi tempus genitale diemque

primigenum maris et terrae solisque coortum

addita corpora sunt extrinsecus, addita circum

semina, quae magnum iaculando contulit omne, unde mare et terrae possent augescere et unde appareret spatium caeli domus altaque tecta

tolleret a terris procul et consurgeret aer.

Nam sua cuique, locis ex omnibus, omnia plagis corpora distribuuntur et ad sua saecla recedunt, umor ad umorem, terreno corpore terra crescit et ignem ignes procudunt aetheraque 〈aether), donique ad extremum crescendi perfica finem omnia perduxit rerum natura creatrix; ut fit ubi nihilo iam plus est quod datur intra vitalis venas quam quod fluit atque recedit. Omnibus hic aetas debet consistere rebus, hic natura suis refrenat viribus auctum. Nam quaecumque vides hilaro grandescere adauctu paulatimque gradus aetatis scandere adultae, plura sibi assumunt quam de se corpora mittunt, dum facile in venas cibus omnis inditur et dum non ita sunt late dispessa, ut multa remittant et plus dispendi faciant quam vescitur aetas. Nam certe fluere atque recedere corpora rebus multa manus dandum est; sed plura accedere debent, donec alescendi summum tetigere cacumen. Inde minutatim vires et robur adultum frangit et in partem peiorem liquitur aetas. Quippe etenim quanto est res amplior, augmine adempto, et quo latior est, in cunctas undique partis plura modo dispargit et a se corpora mittit, nec facile in venas cibus omnis diditur ei nec satis est, proquam largos exaestuat aestus, unde queat tantum suboriri ac subpeditare. Iure igitur pereunt, cum rarefacta fluendo sunt et cum externis succumbunt omnia plagis, quandoquidem grandi cibus aevo denique defit, nec tuditantia rem cessant extrinsecus ullam corpora conficere et plagis infesta domare. Sic igitur magni quoque circum moenia mundi expugnata dabunt labem putris〈que〉 ruinas. Omnia debet enim cibus integrare novando et fulcire cibus, 〈cibus〉 omnia sustentare, nequiquam, quoniam nec venae perpetiuntur 
quod satis est, neque quantum opus est natura ministrat.

Iamque adeo fracta est aetas effetaque tellus

1150

vix animalia parva creat, quae cuncta creavit

saecla deditque ferarum ingentia corpora partu.

Haud, ut opinor, enim mortalia saecla superne

aurea de caelo demisit funis in arva

nec mare nec fluctus plangentis saxa crearunt,

1155

sed genuit tellus eadem quae nunc alit ex se.

Praeterea nitidas fruges vinetaque laeta

sponte sua primum mortalibus ipsa creavit,

ipsa dedit dulcis fetus et pabula laeta;

quae nunc vix nostro grandescunt aucta labore,

1160

conterimusque boves et viris agricolarum,

conficimus ferrum vix arvis suppeditati:

usque adeo parcunt fetus augentque laborem.

Iamque caput quassans grandis suspirat arator

crebrius, in cassum magnos cecidisse labores,

et cum tempora temporibus praesentia confert praeteritis, laudat fortunas saepe parentis.

Tristis item vetulae vitis sator atque (vietae) temporis incusat momen saeclumque fatigat, et crepat, antiquum genus ut pietate repletum perfacile angustis tolerarit finibus aevum, cum minor esset agri multo modus ante viritim. Nec tenet omnia paulatim tabescere et ire ad capulum spatio aetatis defessa vetusto.

1110 appareret $L$ : appariret $O Q \quad 1115$ aether add. I 31 : aeraque aer Lachmann 1116 extremum (cf. i. 555) extremam Lachmann 1120 hic Christ: his OQ hilaro Lambinus : hilar OQV : hilari Avancius 1124 corpora $Q^{1}$ : cora $O Q \quad 1126$ dispessa Munro : dispersa OQV 1129 debent $I 31$ : debet OQV 1136 diditur Marullus : deditur OQV 1138 queat Marullus : queant $O Q V$ ante 1139 versus 1146-9 collocavit Goebel 1145 que add. $Q^{1}$ 1147 cibus add. Vossius 1150 fracta $Q^{1}$ : facta $O Q V$ effeta $O^{1} Q$ : effecta $O 1153$ enim mortalia Gellius : et immortalia $O$ : e inmortalia $Q V \quad 1162$ suppeditati] suppetiati Ellis : suppeditat iam Christ, qui post conficimus interpunxit 1163 laborem Pius : labore OQV 1165 magnos $Q^{1}$ : magnum $O Q$ : manuum Vossius labores] laborem $[P]$ ante 1168 versus 1170-2 collocavit Bergk 1168 vietae Heinsius : fatigat OQV (ex 1169) : caducae Merrill 1169 momen Pius : nomen OQV saeclum] caelum Wakefield 1174 capulum : Vossius : scopulum $O$ : copulum $Q$

Dopo il tempo nativo del mondo e il sorgere del primo giorno 1105 del mare, della terra e del sole, molti corpusculi primordiali s'aggiunsero dall'esterno e particelle seminali sopravvennero all'intorno, addensandosi scagliate dall'immenso universo, da cui potessero accrescersi il mare e le terre, e la dimora del cielo acquistasse nuovo spazio elevando lontano da terra i suoi alti tetti, e l'aria si innalzasse. Infatti da tutte le parti tutti i corpi sospinti dagli urti 
si distribuiscono disponendosi ciascuno nella propria specie, l'acqua va nei liquidi, la terra s'accresce di sostanza terrena, i fuochi modellano la fiamma, i semi eterei l'etere,

finché l'industre terra, creatrice delle cose,

fa giungere tutte le essenze all'estremo limite della crescita, come accade quando nulla penetra nelle vene vitali in misura di ciò che ne fluisce e si allontana.

Qui deve arrestarsi l'età di tutte le cose, qui la natura con le sue forze frena ogni crescita. Infatti tutte le creature che vedi ingrandirsi con lieto slancio, e a poco a poco salire i gradini dell'età adulta, assorbono in sé più corpuscoli di quanti ne perdono, finché il cibo si distribuisce facilmente in tutte le vene, ed esse non sono così largamente espanse da emanare numerosi elementi, e da fare maggiore dispendio di quanto la loro età non assimili. È infatti una realtà certa che le particelle elementari fluiscono e si allontanano dai corpi: ma più devono aggiungersi finché non siano pervenute al vertice della crescita.

Poi a grado a grado l'età infrange le forze e l'adulta energia, e piega verso il proprio declino.

Venuto meno lo sviluppo, quanto un corpo è più grande

e più vasto, tanti più semi germinali emette da sé e disperde ovunque in tutte le direzioni;

il cibo non si diffonde facilmente in tutte le vene, e non è sufficiente in rapporto a quanto fluisce a larghi fiotti, a poter riformarne altrettanti e a riparare le perdite.

Giustamente dunque le cose periscono quando estenuate dal deflusso soccombono tutte agli urti esterni, poiché in vecchiaia infine il cibo viene a mancare e i corpuscoli martellanti dall'esterno non cessano di stremare alcuna cosa e di vincerla ostili con gli urti.

Così dunque anche le mura del vasto mondo espugnate d'attorno crolleranno corrose in rovina.

Ogni cosa dev'essere infatti ristorata dal cibo che la rinnovi, e sostenuta da esso: tutto dev'essere sostentato dal cibo, ma invano, perché con il tempo le vene non sopportano più quanto basti alla vita, né la natura appresta il necessario. Ormai la nostra età è stremata, la terra esausta produce a stento meschini esemplari, la terra che un giorno generò ogni specie e creò dal suo grembo animali dai corpi possenti. Non certo una fune d'oro pendula dal cielo, io credo, calò in terra dall'alto le stirpi mortali né queste furono generate dal mare e dai flutti che s'infrangono sugli scogli, bensì dalla stessa terra che ora di sé le alimenta. Inoltre essa in principio creò le splendide messi e i vigneti rigogliosi per sua forza spontanea in favore dei mortali, essa donò i dolci frutti e pascoli lieti, 
che ora crescono a stento aiutati dalla nostra fatica, 1160 e stremiamo i buoi e tutte le forze dei contadini, e logoriamo il ferro, appena sostentati dai campi: a tal punto sono scarsi di frutta e accrescono la nostra fatica. Ormai scuotendo il capo il vecchio aratore sospira più spesso, vedendo le proprie grandi fatiche cadute nel nulla, e quando paragona il tempo presente al passato, non può che elogiare di frequente la fortuna del padre. E anche il mesto colono della vecchia vigna avvizzita accusa il corso del tempo e impreca all'età, brontolando che gli antichi, ricolmi di buone virtù, trascorrevano una vita estremamente più agevole in modesti poderi, essendo di molto minore la parte di terra di ognuno, e non pensa che tutto man mano rovina e si avvia a morte consunto dal lungo spazio di tempo.

\subsection{Introduzione}

Lucrezio affronta di nuovo il tema della mortalità del mondo al culmine della sezione conclusiva del secondo libro. Ė il poeta stesso a segnare una cesura netta rispetto agli argomenti affrontati nel resto del libro, mediante un ampio appello al lettore (vv. 1023-1047), invitato a non essere spaventato dalla novitas delle dottrine che stanno per essere esposte, ma, piuttosto, a valutarle senza un rifiuto preventivo. ${ }^{1}$ Si noti che Lucrezio ripropone implicitamente il modello del trionfo di Epicuro in 1.62-79:2 egli infatti esorta il lettore a proiettare la mente nello spazio immenso extra moenia mundi (vv. 1045-1047) sino a un'illuminazione della reale natura dell'universo (v. 1051). ${ }^{3}$ Una volta riaperta la porta sull'infinito, il poeta può esporre la dottrina della pluralità dei mondi (vv. 1048-1089), ${ }^{4}$ alla

1 I contenuti di questo passo saranno esaminati alle pp. 268-270.

2 Tale modello è già operante nei vv. 1040-1042 desine [...] novitate exterritus ipsa / exspuere ex animo rationem, sed magis acri iudicio perpende; cfr. 1.68-70 quem neque fama deum nec fulmina nec minitanti / murmure compressit caelum, sed eo magis acrem / irritat animi virtutem.

3 A proposito della discussa espressione animi iactus liber in 2.1047 cfr. Schrijvers 2007, 277-278 e Sedley 2018, 155-160.

4 La spiegazione offerta consta di tre argomenti: dati l'infinità dell'universo e l'immenso numero di atomi, è impossibile che non esistano altri mondi (vv. 1048-1066); la natura è sempre la medesima, in ogni luogo e in ogni tempo, pertanto mai vengono meno le precondizioni che permettono l'esistenza di altri mondi (vv. 1067-1076); il principio dell'isonomia impone che non esista mai un solo esemplare di una specie animale. Tale principio è valido anche per i pianeti (vv. 1077-1089). Su quest'ultimo punto, cfr. la nota affermazione del democriteo Metrodoro di 
quale segue una parentesi anti-provvidenzialistica, volta a negare che degli dèi onnipotenti e ubiqui governino l'universo (vv. 1090-1104). ${ }^{5} \mathrm{Si}$ apre poi, finalmente, la vera e propria conclusione (vv. 1105-1174): qui il poeta ripercorre in modo rapido le tappe dello sviluppo del nostro mondo (e di ogni altro mondo esistente o esistito nell'universo): cosmogonia, accrescimento, acme, declino e disgregazione. Tale percorso è descritto mediante il modello analogico del $\mu \alpha \kappa \rho \alpha ́ v \theta \rho \omega \pi о \varsigma$, secondo il quale il cosmo è strutturato e funziona come un corpo umano: ${ }^{6}$ la crescita del mondo è infatti paragonata a quella di un essere vivente che diviene adulto grazie all'afflusso del cibus atomico nelle sue vene; alla maturità segue però inevitabilmente la vecchiaia, che si chiude con il crollo dei moenia mundi, orma corrosi dal tempo e abbattuti dal medesimo afflusso di atomi che prima li aveva nutriti (vv. 1144-1145). I versi finali (vv. 1150-1174) si focalizzano sul tema dell'invecchiamento della terra, giunta quasi alla sterilità e capace di produrre raccolti soltanto grazie alla fatica di uomini e animali. Nei vv. 1164-1174, cui si rivolgerà particolare attenzione nell'Appendice 1, tale drammatica situazione è presentata dalla prospettiva di due vecchi contadini romani, incapaci di comprendere l'inesorabile legge della vetustas. Svolte queste premesse, è ora possibile procedere all'analisi dei vv. 1105-1174, prendendo di nuovo le mosse dalle loro principali interpretazioni proposte dalla critica lucreziana.

\subsection{Solmsen: Lucrezio come fedele traduttore di Epicuro}

L'analisi delle fonti del finale del secondo libro lucreziano deve trarre inizio dall'esaustivo studio svolto da Solmsen. ${ }^{7}$ Per prima cosa, questi sottolinea l'importanza del modello costituito da un passo di Democrito, già citato nell'introduzione, nel quale si percorre lo sviluppo di un cosmo fino al momento in cui esso non è più in grado di accogliere qualcosa dall'esterno. ${ }^{8}$ A parere dello studioso, i versi di Lucrezio, seppur in relazione con il passo democriteo, pre-

Chio, secondo il quale l'esistenza di un solo mondo nell'universo sarebbe tanto inverosimile quanto una sola spiga di grano in un campo (cfr. D.-K. 70 A6).

5 Cfr. pp. 95-97.

6 Una breve anticipazione di questo modello analogico si ritrova già in 1.1038-1041: qui Lucrezio, per spiegare le modalità della crescita e della dissoluzione del mondo, compara l'afflusso e la fuga degli atomi dal cosmo al nutrimento di un essere vivente (nam veluti privata cibo natura animantum / diffluit amittens corpus, sic omnia debent / dissolvi).

7 Cfr. Solmsen 1953, 33-51.

8 D.-K. 68 A40. 
sentano tuttavia una teoria più elaborata, dovuta con ogni verosimiglianza alla revisione di essa compiuta da Epicuro. ${ }^{9}$ Tale teoria compara infatti il corpo del mondo a un corpo umano, assegnando un ruolo chiave alle vene, che distribuiscono il nutrimento e garantiscono la crescita (cfr. v. 1119; v. 1125); solo la rottura di tale equilibrio provoca la dissoluzione dell'organismo (cfr. v. 1136; vv. 1148-1149). Solmsen si volge poi ad analizzare le corrispondenze tra la dottrina biologica epicurea esposta da Lucrezio e applicata al divenire del mondo e alcuni passi platonici dal Timeo, relativi al sostentamento del corpo umano tramite le vene, sempre secondo un principio isomorfico. ${ }^{10} \mathrm{Il}$ quadro generale del nutrimento e dello sviluppo corporeo fornito da Platone è ritenuto dallo studioso sostanzialmente identico a quello del passaggio lucreziano. ${ }^{11}$ Pur non escludendo in toto la possibilità una ripresa polemica del Timeo da parte di Epicuro, Solmsen ritiene però molto più probabile l'ipotesi che tale «coherent and consistent theory of nutrition, growth and decay» fosse semplicemente uno dei capisaldi della scienza medica nel IV secolo. ${ }^{12}$ Richiamando il fr. 305 Usener, lo studioso riconduce a Epicuro l'applicazione sistematica e definitiva al cosmo di tale teoria, prima riferita al corpo umano. ${ }^{13}$ In sintesi, il «biological pattern of growth and decline» sotteso all'argomento dell'invecchiamento del mondo non costituirebbe una novità lucreziana: ${ }^{14}$ al contrario, l'intera sezione conclusiva

9 Cfr. Solmsen 1953, 37 n. 15: «clearly, this revision of a Democritean tenet is of a piece with other refinements that Epicurus felt compelled to make. Nor is the motive for this particular refinement hard to find. It suggested itself as result of the parallel between the Cosmos and an organic body which Epicurus keeps up throughout his argument».

10 Cfr. le cosiderazioni di Solmsen 1953, 42 a proposito di Pl. Ti. 78e-81d: «here we read how the food (after having been cut up in the belly) is "pumped" into the blood-vessels [...]. As long as the body is "young" the food assimilitad surpasses in quantity the matter given off to elements; later on, in the years of decline of yñ $\rho \alpha$, the relationship is reversed until in the end the food supply is altogether inadequate to counteract the never-ceasing attacks from the outside and the body finally succumbs».

11 Si confronti in particolare la descrizione dell'invecchiamento e della consunzione del mondo, dovuti all'impatto degli atomi provenienti dall'esterno in Lucr. 2.1122-27 e 1148-1149 con l'esposizione delle dottrine relative alla degenerazione del corpo in Pl. Ti. 81a4- d4.

12 Cfr. Solmsen 1953, 39: «we have no way of knowing how long before the Timaeus and by whom as the first the formula was developed. To understand its bearing we must remember that in some of the late Presocratic systems the Cosmos too grows as long as it grows by the addition of like to like, i.e. in the same fashion as human beings, animals and plants».

13 Cfr. Solmsen 1953, 48.

14 Cfr. Solmsen 1953, 42: «if we consider the passage in the light of its antecedents, we have no choice but to regard the cibus and the venae of the world as part and parcel of Epicurus' arguments. What he did was to transfer the current theory about nutrition, growth and decline from biology to cosmology, from the Microcosm to the Macrocosm». 
del secondo libro del De rerum natura rappresenterebbe una fedele ripresa delle parole di Epicuro. ${ }^{15}$

\subsection{Schrijvers: Lucrezio come "eclettico epigono dell'età tardo ellenistica"}

Ponendosi su posizioni diametralmente opposte a quelle di Solmsen, ${ }^{16}$ Schrijvers evidenzia una serie di analogie e parallelismi tra alcuni passi del De rerum natura (compreso il nostro finale) e alcuni passi appartenenti a testi tardoellenistici fortemente influenzati dal pensiero peripatetico, ovverosia il trattato pseudo-aristotelico De mundo, ${ }^{17}$ il De universi natura (attribuito al neopitagorico Ocello Lucano $)^{18}$ e il De aeternitate mundi di Filone Alessandrino. Tali parallelismi dimostrerebbero una ripresa di questi trattati da parte del poeta romano, capace, come gli eclettici epigoni della sua epoca, di sfruttare in senso epicureo dottrine appartenenti a scuole avverse. ${ }^{19}$ La serie di parallelismi che interessa l'analisi del finale del secondo libro concerne in particolare uno di questi trattati, ovverosia il De aeternitate mundi: nei capp. 55-75, infatti, Filone riporta alcune dottrine attribuite al peripatetico Critolao a proposito dell'eternità del mondo e, soprattutto, dell'eterna giovinezza della terra, in opposizione a quanti ne asseriscono il progressivo invecchiamento. Schrijvers evidenzia una relazione diretta tra gli argomenti di Critolao e quelli del finale del secondo libro lucreziano, nonché di un altro passo del De rerum natura (5.807-836) dove Lucrezio

15 A prova di tale lettura, Solmsen 1953, 51 asserisce infine che non è un caso che l'argomento di retaggio "presocratico" dell'invecchiamento del mondo trovi spazio nel finale del secondo libro e non nel quinto libro (vv. 235-415), in cui questi argomenti «show a definitive emancipation from the traditional pattern which our section in book II revises».

16 Cfr. Schrijvers 1978, 77-114. Si farà riferimento alla traduzione inglese di questo lavoro (Schrijvers 2007, 255-288).

17 Per un'introduzione al rapporto tra De mundo e De rerum natura, cfr. Appendice 4.

18 Cfr, pp. 18-19.

19 Cfr. e.g. Schrijvers 2007, 266, in riferimento alla presenza nel De rerum natura di passi che sembrano contenere riprese del luogo comune ellenistico delle laudes terrae (sul quale cfr. anche Landolfi 1985): «this contention is congruent with the characterization of Lucretius as an eclectic epigone which emerged from the parallels between the De rerum natura, the De mundo, and the De aeternitate mundi outlined above. The eclectic and dialectic interplay in which philosophical opponents borrow themes, arguments, figures of thought, and also root metaphors from one another, sometimes in order to turn them against the school in which they originated, is characteristic of the late Hellenistic era». 
riprende il tema della Madre-terra e la questione della generazione dei primi esseri viventi. ${ }^{20}$

Pur dichiarando impossibile stabilire con esattezza quale sia la relazione tra Filone Alessandrino, Critolao e Lucrezio, Schrijvers asserisce che i parallelismi da lui evidenziati dimostrano la presenza di un legame certo tra Lucrezio e i trattati sopra citati, ben superiore a una generica consonanza tematica. ${ }^{21}$ Pertanto, Lucrezio si dimostrerebbe a tutti gli effetti «autore eclettico della tarda età ellenistica», libero da vincoli di totale fedeltà a Epicuro. Non da quest'ultimo

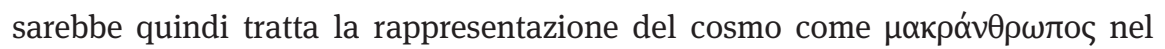
finale del secondo libro, bensì attinta a quella serie di temi filosofici e motivi topici che costituivano il Bildungsgut della tarda età ellenistica. ${ }^{22}$

Questa interpretazione, secondo Schrijvers, spiegherebbe inoltre le numerose contraddizioni in cui Lucrezio sembra incorrere utilizzando modelli analogici biologici («biological illustrations») come Leitmotiv nel primo e nel secondo libro. Tali contraddizioni sarebbero dunque motivate dalla ripresa sistematica, da parte di Lucrezio, dei trattati tardo-ellenistici sopra citati: questi testi risultano infatti fortemente influenzati dalla filosofia peripatetica e da una serie di motivi e luoghi comuni della tarda età ellenistica («commonplace of Hellenistic thought»), incompatibili con il pensiero epicureo. ${ }^{23}$

20 Un altro importante punto di contatto tra De rerum natura e De aeternitate mundi richiamato da Schrijvers concerne la rappresentazione della crescita del mondo come una sorta di salita sino a un punto "apicale" (Lucr. 2.1122-30; Philo aet. 58). Tuttavia, Schrijvers si concentra soprattutto sul V libro del De rerum natura, dove troviamo notevoli paralleli con le dottrine riportate da Filone: in particolare, la rappresentazione della Madre-Terra come una giovane donna incinta, capace di generare da sé i primi uomini e poi nutrirli con fiumi di latte (Lucr. 5.807-836; Philo aet. 58-68).

21 Cfr. Schrijvers 2007, 269-270: «the quality of the parallels listed above nevertheless permits us, in my view, to assume that Lucretius, as an eclectic author of the late Hellenistic era, constructed his work as a composite, drawing on the reservoir of philosophical and scientific themes of the schools which made up the Bildungsgut of his age».

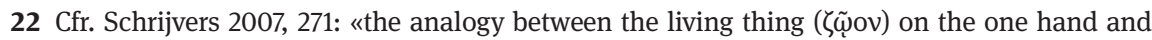
the cosmos or the earth on the other is found only twice in Epicurus: once in a doxographic notice, and once, in very concise form, in a fragment from Book 11 of On Nature. In my opinion, it is not legitimate to deduce from these two texts that the analogical image of the world as

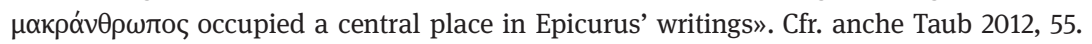

23 Schrijvers 2007, 265: «strictly speaking, the theory that matter is imperishable [...] should not be illustrated within the framework of Epicurean physics by the $\mu \varepsilon \tau \dot{\alpha} \beta \alpha \sigma \iota \varsigma, ~ \varepsilon ́ \xi \dot{\alpha} \lambda \lambda \hat{n} \lambda \omega \nu$ of plant and animal life on earth, since this earth and the living things which populate it have been born and are destined to die». 


\subsection{Schiesaro: il ritorno alla tesi di Solmsen}

Più recentemente, Schiesaro ha svolto una critica profonda delle tesi di Schrijvers, evidenziando come il quadro finale del secondo libro (così come la sezione relativa alla "primavera del mondo" nel quinto) dimostri un'ossatura «strettamente epicurea». ${ }^{24}$ Pertanto, Lucrezio non potrebbe essere etichettato assolutamente come un abile eclettico, che costruisce il proprio discorso alla maniera di un collage di teorie eterogenee rintracciabili nella letteratura a lui contemporanea. Schiesaro mette infatti in rilievo come numerosi temi e motivi topici che Schrijvers attribuisce al Bildungsgut tardo-ellenistico debbano in verità essere fatti risalire all'età classica o all'età arcaica. Secondo lo studioso, è parimenti da recusare l'idea di Schrijvers secondo la quale «dall'interazione tra un certo explicans e l'explicandum si produce una serie di associazioni indebite, che proietta sul secondo caratteristiche che gli sono estranee, che contrastano con l'ortodossia epicurea, e che si ricollegano, piuttosto, a dottrine allotrie». ${ }^{25}$

Lo studioso prende le mosse dal modello analogico del $\mu \alpha \kappa \rho \alpha ́ v \theta \rho \omega \pi о \varsigma,{ }^{26}$ sfruttato da Lucrezio nonostante la sua apparente inconciliabilità con la dottrina epicurea che ritiene il cosmo mero aggregato inanimato di atomi e vuoto. ${ }^{27}$ L'uso di questo modello analogico da parte di Lucrezio non costituisce, secondo Schiesaro, un allontanamento dall'ortodossia epicurea: riprendendo Solmsen, egli mostra come tale modello fosse già diffuso nelle cosmologie presocratiche. ${ }^{28}$ Al contempo, Schiesaro evidenzia la «cautela» con cui Lucrezio utilizza il modello del $\mu \alpha \kappa \rho \alpha ́ v \theta \rho \omega \pi$, prova del fatto che il poeta fosse ben conscio «degli eventuali pericoli di ambiguità» associati all'uso di esso: da questa consapevolezza deriva un'estrema cura nel limitare ogni margine di confusione e ogni rischio di eterodossia. ${ }^{29}$

24 Cfr. Schiesaro 1990a, 107-108: «non è vero che Lucrezio dipende dal retroterra culturale a lui contemporaneo, ma l'operazione del poeta sembra invece quella di riprendere la tesi epicurea, ripresentandola in versi, con particolare ricerca di efficacia».

25 Schiesaro 1990a, 73.

26 Schiesaro 1990a, 74.

27 Schiesaro 1990a, 74: «l'archetipo del mondo come grande corpo umano governato dalle stesse leggi e dagli stessi rapporti strutturali viene prospettato nel De rerum natura in riferimento a diversi problemi, i cui principali sono la crescita, il declino, la dissoluzione del nostro mondo - esemplare di tutti gli altri mondi - in termini di evoluzione biologica, e il chiarimento dei rapporti di interdipendenza e organicità delle varie parti del mondo per confronto con l'organizzazione del corpo umano».

28 Cfr. Schiesaro 1990a, 75 e Sedley 2011, 26-28.

29 Cfr. Schiesaro 1990a, 76: «infatti nei vv. 1118-1122 [...] il confronto tra il mondo e i corpi viventi è istituito concisamente e quasi in parentesi, mentre dal v. 1122 in avanti l'autore estende 
Schiesaro analizza poi i passi del De rerum natura relativi al tema della Terra-madre e della generazione degli animalia riportati da Schrijvers, evidenziando come tali motivi siano in verità già riconducibili ai filosofi presocratici, come ad esempio Empedocle. Lucrezio non dipende quindi «dal retroterra culturale a lui contemporaneo»: la sua operazione si configura invece come fedele «ripresentazione in versi» della tesi epicurea, «con particolare ricerca di efficacia». ${ }^{30}$ Quest'ultima è garantita dalla ripresa di fonti poetiche ben precise, che spaziano dagli Inni omerici alla tragedia di età classica; ${ }^{31}$ notevole l'esempio dei vv. 250-264 del primo libro, che rimandano alla ierogamia del Crisippo euripideo e delle Danaidi eschilee. ${ }^{32}$ Indubbiamente la presenza di queste traduzioni potrebbe cagionare accuse di «disorganicità»: difatti Lucrezio rischia d'esporre a gravi fraintendimenti punti nodali della propria dottrina. Tuttavia Schiesaro sottolinea come anche questi passaggi, nonostante la loro ambiguità, possano essere ricondotti nell'alveo della dottrina epicurea, cosicché, «se perplessità essi suscitano, non per questo dovremo troppo affrettatamente ritenerli una "contraddizione" del poeta con se stesso». ${ }^{33}$

\subsection{Le fonti di Lucrezio: un riepilogo}

Un utile punto di partenza per analizzare le fonti di questo finale lucreziano consiste nel richiamare alcuni dei più importanti passi citati da Solmsen e da Schiesaro. Come si è visto, la storia del cosmo, dalla generazione alla distruzione, preceduta dall'affermazione dell'infinità dell'universo e dell'esistenza di

le sue considerazioni ai corpora in generale, non solo al corpo umano [...]. Il risultato di questa indagine è la definizione di una legge generale sulla crescita e la distruzione dei corpi - viventi e

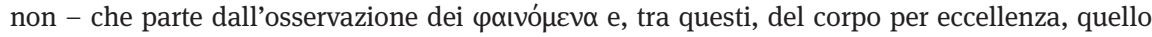
umano». Cfr. anche le considerazioni di Solmsen 1953, 43.

30 Cfr. D.-K. 31 A70, ma si veda anche il Menesseno di Platone (Menex. 237e). Cfr. Schiesaro 1990a, 102; 107: «non è dunque necessario pensare all'utilizzazione, da parte di Lucrezio, di un repertorio tradizionale lui contemporaneo [...] ma che tale associazione possa essere retrodata con ogni verosimiglianza ad Epicuro, e forse anche ai pensatori presocratici».

31 Cfr. Schiesaro 1990a, 108.

32 Cfr. Schiesaro 1990a, 111 e 118.

33 Cfr. Schiesaro 1990a, 120. Si consideri anche 85: «non si dovrà eccedere, invece, nel sottolineare tentazioni eclettiche o scopertamente eterodosse di Lucrezio: anche nei casi in cui talune implicazioni delle analogie o delle metafore di base sembrano non accordarsi del tutto con alcuni principi della dottrina epicurea, si può ricostruire con sufficiente sicurezza una analoga presa di posizione dello stesso Epicuro. Il problema dell'eclettismo di Lucrezio, quindi, si trasforma se mai in quello dell'eclettismo di Epicuro». 
infiniti mondi, si ritrova già in un frammento di Leucippo. ${ }^{34}$ Questo passo presenta in comune con i versi lucreziani il salto argomentativo dal tema dell'infinità dell'universo (

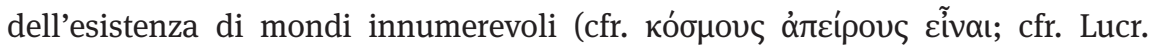
2.1048-1089) e, infine, la narrazione della cosmogonia, dovuta all'afflusso di

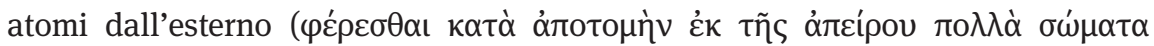

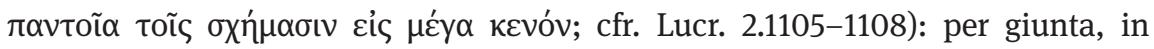
entrambi i testi si specifica che gli atomi si raggruppano secondo un principio

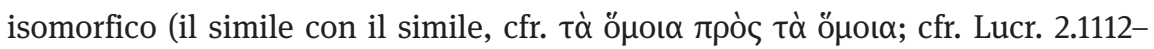
1115). Infine, comune è anche l'idea dell'evoluzione del кóouoৎ secondo la se-

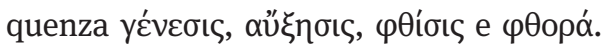

La medesima dottrina viene sviluppata da Democrito, come dimostra il frammento D.-K. 68 A37.35 Alcuni elementi di quest'ultimo dimostrano la correttezza delle argomentazioni di Solmsen e Schiesaro, secondo i quali le radici del modello analogico del $\mu \alpha \kappa \rho \alpha ́ v \theta \rho \omega \pi о \varsigma$ debbono essere ricondotte alla filosofia presocratica. A questo proposito, notevole è il nesso tra l'esistenza degli

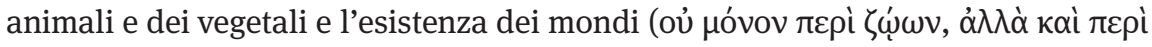

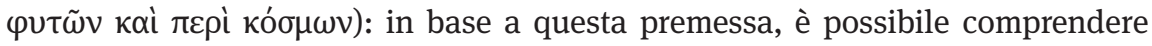

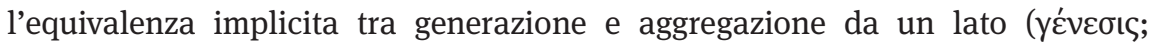

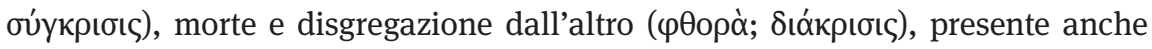
nel passo lucreziano (cfr. 2.1104 mundi tempus genitale e 2.1144 dabunt labem putrisque ruinas). Analoghe considerazioni possono essere svolte a proposito del frammento democriteo D.-K. 68 A40 (= Hippol. Refut. I 13):

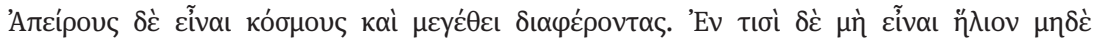

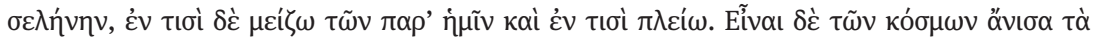

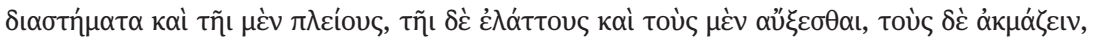

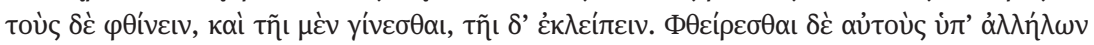

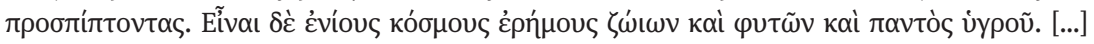

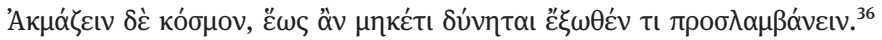

34 Cfr. D.-K. 67 A1 (= 289 Luria). Parte dei passi qui citati sono oggetto di commento alle pp. 3-5. 35 D.-K. 68 A37.

36 «I mondi sono infiniti e diversi per grandezza, cosicché in alcuni non esistono né sole né luna, in altri ve ne sono di più grandi che nel nostro cosmo, e in altri ancora ce ne sono più numerosi. Gli intervalli che separano i mondi sono differenti, cosicché da una parte ci sono più mondi e da un'altra meno, e alcuni si ingrandiscono, mentre altri sono all'apice dell'ingrandimento. Altri, poi, si rimpiccioliscono, cosicché da una parte si originano nuovi cosmi e dall'altra scompaiono. I mondi si corrompono nel collasso di uno con l'altro. Alcuni mondo non hanno animali, piante e perfino umidità. [...] Un mondo si sviluppa fintanto che non può accogliere niente altro dall'esterno». 
Anche questo passo presenta numerose analogie con quello leucippeo sopra citato e ulteriori punti di tangenza con il finale lucreziano. In riferimento a quest'ultimo, gli elementi più degni di nota sono:

- Il passaggio logico dalla teoria dell'esistenza di mondi innumerevoli alla descrizione del loro percorso di crescita e di dissoluzione (già presente in Leucippo).

- La descrizione “organicistica” dell'evoluzione del mondo, suddivisa in ge-

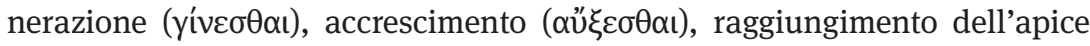

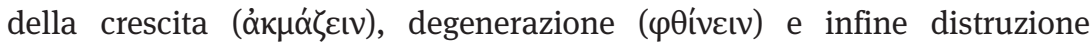
( $\varphi \theta \varepsilon i ́ \rho \varepsilon \sigma \theta \alpha \iota)$.

- La definizione del momento apicale come situazione in cui un mondo non

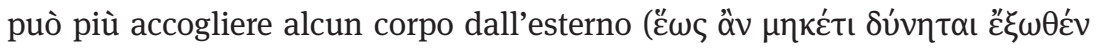

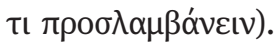

Alla ók $\mu$ ń democritea, momento di massima capacità assimilativa prima della decadenza, corrisponde in Lucrezio il momento dell'extremus crescendi finis, ovverosia la fase nella quale un mondo raggiunge un perfetto (ma effimero) equilibrio nei suoi scambi atomici con lo spazio esterno. Non si dimentichi infine che a Democrito può essere ricondotta anche la tematica dell'invecchiamento e del progressivo isterilimento della terra, definita in alcuni passi come ormai incapace di generare esseri viventi di grandi dimensioni. ${ }^{37}$ Come si è visto, anche questo elemento è attestato nel finale lucreziano (vv. 1151-1152): vix animalia parva creat, quae cuncta creavit / saecla deditque ferarum ingentia corpora partu.

Più complesso è ritrovare informazioni relative al racconto epicureo a proposito dell'invecchiamento del mondo e del progressivo isterilimento della terra. Un utile punto di partenza è sicuramente l'Epistola a Pitocle (88-90):

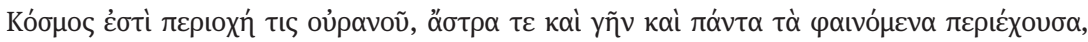

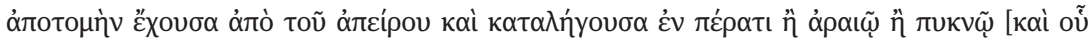

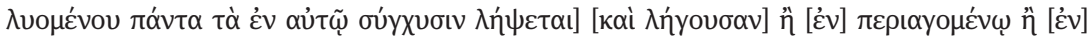

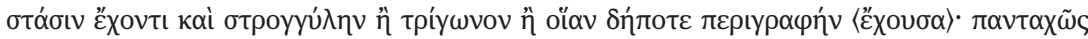

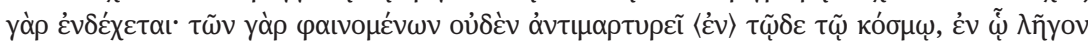

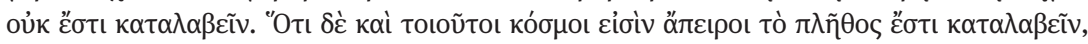

37 Cfr. D.-K. 68B5.2 (= Hermippus de astrol. [Ioann. Catrares] 2.1.4ff. p. 33.15 Kroll-V.) tò yà

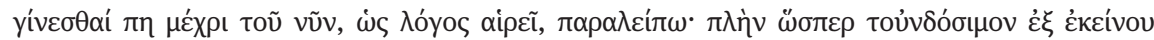

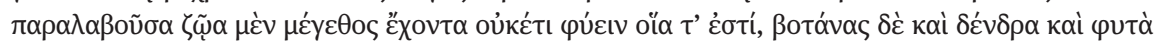

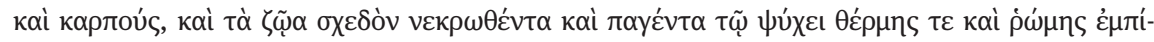
$\pi \lambda \alpha \tau \alpha$. Per un commento a questo passo, cfr. Enriques-Mazziotti $2016^{2}$, 272-277. Si noti che

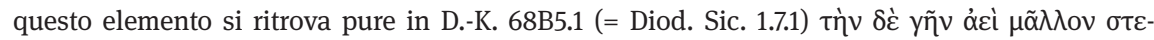

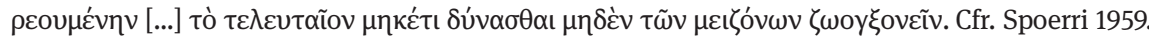




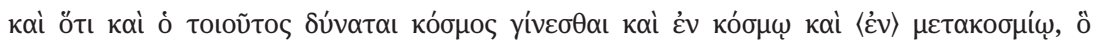

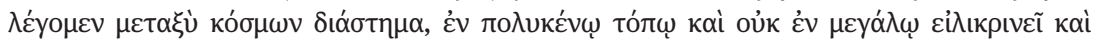

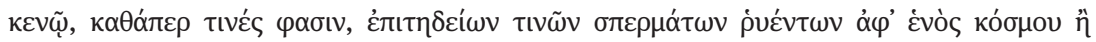

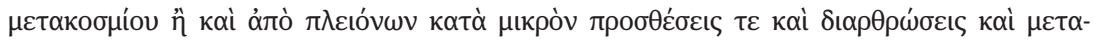

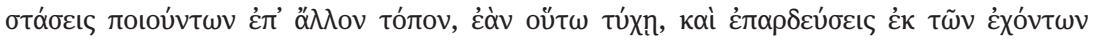

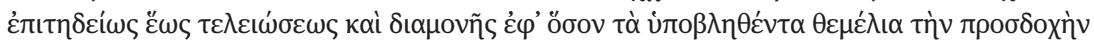

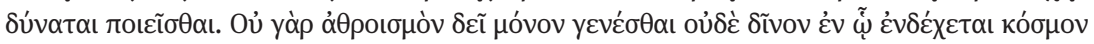

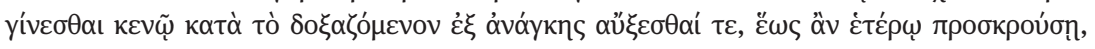

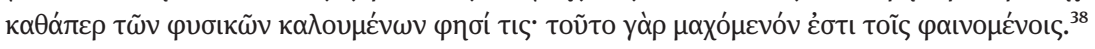

Come nei passi degli Atomisti, troviamo qui il passaggio argomentativo dalla

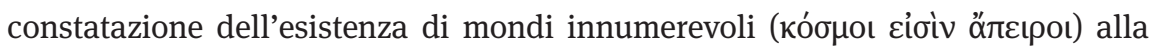
descrizione della loro formazione, dovuta all'afflusso di atomi dall'esterno. Riguardo poi allo sviluppo di ciascun cosmo, il filosofo si limita a notare che vi è

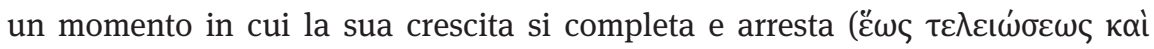

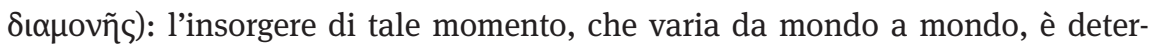

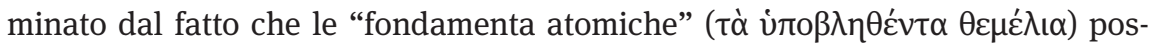
sono sopportare solo una quantità limitata di aggiunte, prima dell'inevitabile crollo. Quest'ultimo è descritto, in un scolio al cap. 88, come un processo di dissoluzione, che causerà la rovina e la confusione di ciascuna delle componenti

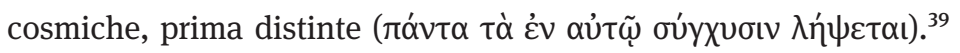

38 «Un mondo è una determinata porzione circoscritta di cielo, contenente astri e terra e tutte le cose sensibili, che ha netta separazione dall'infinito e termina in un limite poroso o fitto, e la cui dissoluzione produrrà la rovina di tutto ciò che contiene. $\mathrm{E}$ che poi siffatti mondi siano infiniti di numero si può determinare con l'intelletto; come pure si può determinare che un simile mondo può prodursi o in un mondo o in un intermundio (così chiamiamo l'intervallo tra due mondi); in uno spazio che sia molto vuoto, ma non però in un grande spazio assolutamente privo di materia e vuoto, come vogliono alcuni. E si produce, quando determinati atomi acconci vi affluiscono, da un solo o più mondi od intermondi. Tali atomi, a poco a poco, vi si aggiungono, si connettono, o anche si trasferiscono da un luogo all'altro, a seconda dei casi, e successivamente vi affluiscono da nuclei di materia acconcia, fino a che raggiuga il compimento e l'arresto del suo crescere, per quanto le basi sottoposte permettono aggiunta di materia. Non basta infatti che avvenga solo un accozzo di atomi ed un moto vorticoso, in quel vuoto in cui, secondo quanto si crede, è possibile che un mondo si crei per necessità meccanica e che cresca fino a che s'urti con un altro, come affermano alcuni di quelli che sono chiamati fisici. Poiché questo è in contrasto con i fenomeni».

39 Le parole finali di questo passo epicureo (cap. 90), svolte in chiara polemica con la cosmologia democritea (cfr. pp. 3-5 di questo lavoro), sembrano confermare le considerazioni di Solmsen a proposito del fatto che Epicuro avesse operato una sorta di aggiornamento delle dottrine atomistiche, riformulate secondo i principi del nuovo sapere scientifico tra IV e III secolo a.C. Sulla contrapposizione tra gli Atomisti ed Epicuro a proposito della dottrina del vortice (connesso al concetto di "necessità"), cfr. Perilli 1996, 87-101. 


\subsection{Lucrezio e le teorie peripatetiche esposte in Philo aet. 55-75}

Il confronto tra il finale del secondo libro del De rerum natura e i passi citati evidenzia che Lucrezio non sembra "tradire" lo schema di evoluzione del mondo e della terra elaborato dagli Atomisti e sviluppato da Epicuro. Tale constatazione non può però indurre a concludere che il poeta si limiti a tradurre le dottrine lette nel trattato Sulla natura. Un'attenta lettura delle fonti epicuree a noi pervenute mostra infatti che Lucrezio seleziona con attenzione le tematiche da trattare, spesso omettendo (o, talvolta, solo accennando) numerosi punti che invece sembrano fondamentali agli occhi di Epicuro. ${ }^{40}$

Il primo esempio notevole della libertà di Lucrezio nei confronti del proprio modello è rappresentato dalla questione delle forme dei mondi. Laddove infatti Epicuro reputa cruciale tale tematica, ${ }^{41}$ il poeta latino decide invece di ometterla. ${ }^{42} \mathrm{Gli}$ argomenti da quest'ultimo dedicati alla dimostrazione dell'esistenza d'infiniti cosmi considerano unicamente quei mondi che appaiono simili al nostro, ovverosia provvisti della stessa struttura e abitati dai medesimi esseri viventi. ${ }^{43}$ Se poi volgiamo lo sguardo alla vera e propria sezione escatologica, notiamo che, ancora una volta, Lucrezio trascura alcuni punti essenziali delle dottrine epicuree: ad esempio, il fatto che alcuni mondi si sviluppano e si dis-

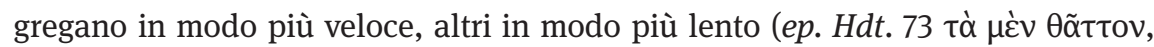
$\tau \alpha \grave{~} \delta \grave{\varepsilon} \beta \rho \alpha \delta$ tepov). Similmente, nel De rerum natura non si ritrova traccia della distinzione tra modalità di dissoluzione operata da Epicuro nel fr. 305 Usener, ${ }^{44}$

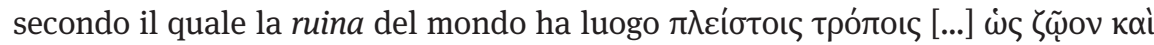

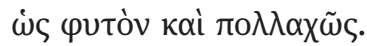

Gli esempi appena riportati mostrano come l'esposizione lucreziana riduca deliberatamente la portata del principio di molteplicità alla base della cosmologia epicurea, che insisteva invece sulla varietà dei mondi, concernente tanto le

40 Come si è visto, Montarese 2018 ha mostrato che tale relativa libertà dalla fonte epicurea sia riscontrabile anche nei vv. 635-920 del primo libro, che non sembrano seguire la traccia offerta dai libri XIV e XV del trattato Sulla natura.

41 Cfr. ep. Hdt. 73-74, con rimando al trattato Sulla natura.

42 Un riferimento fugace al tema della diversità tra i mondi si trova in 5.1345 (in variis mundis varia ratione creatis). Cfr. Luria 2007, 1110-1111 a proposito delle innovazioni apportate da Epicuro su questo tema rispetto alla visione democritea.

43 Cfr. Lucr. 2.1063-1065 necesse est / esse alios alibi congressus materiai, / qualis hic est avido complexu quem tenet aether; 2.1074-1075 necesse est confiteare / esse alios aliis terrarum in partibus orbis / et varias hominum gentis et saecla ferarum. L'assenza di riferimenti a mondi dissimili dal nostro viene notata anche da Sedley 2018, 145-146.

44 Cfr. pp. 16-17. 
loro forme quanto le loro modalità di sviluppo e distruzione. Tale riduzione potrebbe essere motivata dall'intento di mettere in ombra gli aspetti più paradossali e criticabili delle dottrine fisiche del Giardino, facendo leva invece sulle realtà più familiari ai lettori latini. ${ }^{45}$ Ciò potrebbe spiegare anche il ricorso costante al motivo analogico del $\mu \alpha \kappa \rho \alpha ́ v \theta \rho \omega \pi о \varsigma$ che, seppur già presente sina dalla riflessione degli Atomisti, è qui sfruttato in una misura che non pare comparabile a quanto leggiamo negli altri testi epicurei a noi pervenuti. ${ }^{46}$

Pur spingendoci al rifiuto della lettura del finale lucreziano come una fedele traduzione di Epicuro, queste considerazioni non devono però farci ricadere nella tentazione d'interpretare Lucrezio alla maniera di Schrijvers, come un eclettico epigono tardo-ellenistico, talora vicino a posizioni inconciliabili con l'ortodossia epicurea. Su questo fronte, restano valide le riserve avanzate da Schiesaro. È proprio la volontà di attualizzare la ratio del maestro, trasportata in un nuovo contesto e rivolta a un nuovo pubblico, a rendere necessaria l'operazione lucreziana di rielaborazione. Come nel caso del finale del primo libro, una piena comprensione di questo passo può dunque essere raggiunta solo liberando il poeta dalla turris eburnea del suo "fondamentalismo", così da rimettere Lucrezio a contatto con il dibattito filosofico sulla natura del cosmo successivo a Epicuro.

Definito questo quadro interpretativo, è possibile ora rilevare che il lavoro di Schrijvers contiene un'intuizione assai preziosa e degna di approfondimento: come spiegare i punti di tangenza tra il De rerum natura e alcuni trattati peripatetici tardo-ellenistici (De aeternitate mundi, De mundo, De mundi natura)? È possibile determinare contatti tra il De rerum natura e questa trattatistica recente, senza però affermare l'eterodossia o l'eclettismo del poeta? Allo scopo di rispondere a tali domande, i prossimi capitoli mostreranno come vi siano effettive e significative tangenze tra i capitoli 55-75 nel trattato De aeternitate mundi e il finale del secondo libro del poema. ${ }^{47}$ Per prima cosa, pare opportuno

45 Cfr. Sedley 2018, 146. Non si dimentichi che ai vv. 1040-1042 Lucrezio sottolinea proprio l'aspetto della novitas della ratio epicurea agli occhi del pubblico romano e il rischio che essa venga rifiutata con disprezzo.

46 Concordo dunque con Schrijvers, quando afferma (in polemica con Solmsen) che il solo fr. 305 Usener e i pochi accenni provenienti dalle lettere non sono sufficienti per ipotizzare la centralità del $\mu \alpha \kappa \rho \alpha ́ v \theta \rho \omega \pi$ ก nell'opera di Epicuro. Riguardo al ruolo di tale motivo analogico in Lucrezio, cfr. Segal 1998, 72-73.

47 Come si è detto, in verità Schrijvers chiamava in causa non solo il De aeternitate mundi di Filone Alessandrino, ma anche De universi natura di Ocello Lucano e il trattato pseudo-aristotelico De mundo. Sebbene anche questi ultimi testi siano di grande rilevanza, ritengo che, per quanto concerne questo finale lucreziano, l'opera di Filone debba costituire il principale termine di paragone. 
sintetizzare il contenuto di questa sezione del trattato e le sue corrispondenze con il passo lucreziano.

\subsection{La fonte degli argomenti trasmessi da Filone}

I capitoli 55-75 del trattato De aeternitate mundi contengono alcuni argomenti in difesa della dottrina dell'eternità cosmica, attribuiti al peripatetico Critolao. Ancora aperta è la questione relativa a quanto di questa sezione sia realmente ascrivibile al filosofo peripatetico; ${ }^{48}$ ragioni stilistiche e contenutistiche rendono assai probabile che la struttura del passo sia il risultato di una rielaborazione svolta da un autore di età successiva, con lo scopo di corroborare le tesi in difesa dell'eternità del mondo. Gli argomenti che il testo ascrive esplicitamente a Critolao sembrano tre:

- $\quad$ il mondo è eterno perché parimenti eterni sono la terra e il genere umano (capp. 55-57).

- il mondo è eterno perché è esso stesso fonte della propria sussistenza (cap. 70).

- tre sono le cause "interne" della distruzione del mondo: mancanza, malattia, senescenza. Il mondo non è soggetto a nessuna delle tre (cap. 74).

Ciascuna di queste prove dell'eternità del mondo è seguita da altri argomenti:

- critica agli "artefici di miti”, che difendono l'assurdo mito degli Sparti, e a quei filosofi che, in maniera simile, sostengono che la terra, che generò da

48 Per la questione dell'attribuzione a Critolao di questi capitoli filoniani, cfr. Sharples 2010, 176-178 e la sintesi di Sharples 2008, 60: «F. Wehrli included only sections 55 and 70 in his collection of the fragments of Critolaus; certainly the syllogistic form of these sections constrast sharply with the manner of the material in 56-69. H. von Arnim [...] argued that the whole of 5575 came from Critolaus [...]. Against von Arnim, K. Reinhartdt [...] held that some of the arguments are later than Critolaus himself. D.T. Runia [...] argues that there is more from Critolaus than Wehrli allows. There seems no reason to reject Critolaus' authorship of B3-B5 [= cap. 7075], other than the fact that the schematic logical formulation at the start of B1 and B2 is missing in the subsequent arguments, but equally no conclusive reason to assert it». Sebbene la questione non sia qui risolvibile, mi pare fondamentale evidenziare che la tesi qui difesa dell'uso di fonti tardo-ellenistiche da parte di Lucrezio non sarebbe messa in discussione, bensì rafforzata dall'eventuale attribuzione dei capitoli 56-69 ad autori peripatetici successivi a Critolao. A proposito della relazione di quest'ultimo con il mondo latino, non si dimentichi che Critolao, assieme a Carneade e a Diogene di Babilonia, era stato protagonista del noto episodio dell'ambasciata "filosofica" inviata dagli Ateniesi a Roma nel 156/155 a.C. (cfr. e. g. Cic. de orat. 2.155). 
sé il genere umano e lo nutrì con fiumi di latte, sia ora invecchiata e ormai sterile (capp. 58-69).

- critica a coloro che dicono che il mondo è mortale e affermano inoltre che esso è soggetto a crescita, maturità, decadenza e morte. Costoro, infatti, ritengono che il mondo sia anche "razionale" e cadono nell'assurdità d'ipotizzare un mondo privo di logos nella sua infanzia e nella sua vecchiaia (capp. 71-73).

- critica a coloro che affermano che il mondo è mortale, ma accettano l'esi-

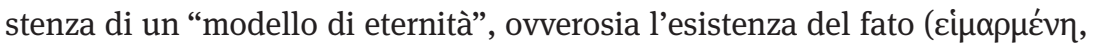
cap. 75).

Gli argomenti appena elencati si contraddistinguono per la notevole vis polemica, diretta contro i nemici dell'eternità cosmica. La diversità di tono rispetto alle più pacate e sillogistiche affermazioni esplicitamente ascritte a Critolao ha fatto sospettare ad alcuni studiosi che questi argomenti debbano essere ricondotti a una fonte diversa dal maestro peripatetico. Si tratta tuttavia di un metodo di attribuzione rischioso, poiché i capitoli 71-75, al di là delle diversità nel tono, sono accomunati da una serrata critica alla cosmologia veterostoica, che ben si accorda alle informazioni che abbiamo riguardo all'appassionato dibattito sollevato da Critolao stesso contro gli Stoici, per giunta su più fronti. ${ }^{49}$

I capitoli 58-69 suscitano indubbiamente più sospetti, perché diretti in primis contro le assurdità insite nel mito degli Sparti, usato come punto di partenza per attaccare quei filosofi che ritengono che i primi uomini siano "germogliati” da uteri radicati nella terra. L'attacco sembra dunque allargare il proprio raggio d'azione, così da includere non solo gli Stoici (comunque assertori della nascita dell'umanità dal suolo, provvidenzialmente "fecondato" dal Logos divino), ${ }^{50}$ ma anche quei pensatori presocratici (da Empedocle agli Atomisti) che avevano teorizzato la nascita del genere umano dalla terra: ${ }^{51}$ né si può escludere che tra gli obbiettivi polemici figuri anche Epicuro, erede di tale visione. Questo dato non deve però spingerci ad attribuire questa ampia sezione al solo Filone: ${ }^{52}$ lo scopo delle argomentazioni qui presentate resta infatti quello di dimostrare l'eternità del genere umano, a riprova dell'eternità del mondo. $\grave{E}$

49 Cfr. Kupreeva 2009, 147-150; Inwood 2014, 51-62. Difatti Sharples 2010, 176-178 attribuisce a Critolao i capitoli 55-57 e 70-75 del trattato filoniano.

50 Si vedano in proposito le fonti richiamate da Dyck 2004, 132.

51 Cfr. Campbell 2003, 77-83.

52 Il segno della rielaborazione di Filone si potrebbe invece individuare nel lessico di certi passaggi e in alcuni poetici ampliamenti; cfr. Sharples 2010, 178 che mette in rilievo «Philo's distinctive affectation in style and vocabulary». 
dunque logico supporre che tali argomenti (o almeno il loro nucleo) possano essere ricondotti a Critolao stesso (come ritenuto da von Arnim) o a una fonte peripatetica successiva..$^{53}$ Definiti questi aspetti, è giunto il momento di rilevare le corrispondenze tra questa sezione del trattato di Filone e il poema lucreziano: - In Lucr. 2.1087-1089 (ma cfr. anche 1.75-77) ${ }^{54}$ le leggi di natura che regolano la crescita e determinano la natura mortale degli esseri viventi, delle specie animali e dei mondi sono rappresentate come irremovibili e immodificabili pietre di confine (vitae depactus terminus alte), conficcate in profondità sin dal principio dei tempi. La medesima immagine ricompare a più riprese nel

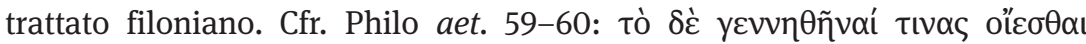

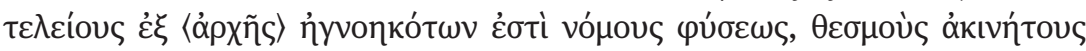

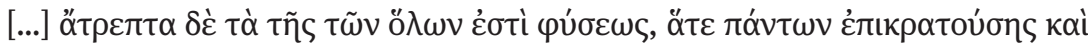

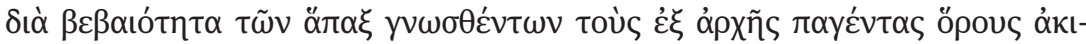

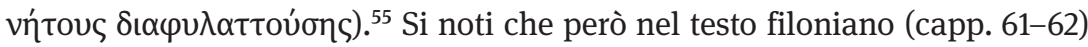
si esclude che anche la terra sia soggetta a tali leggi, poiché essa, come gli

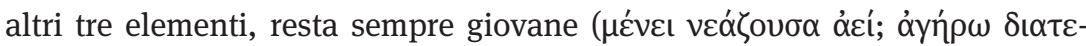
$\lambda \varepsilon \tilde{)}$, contribuendo così, con la sua incorruttibilità, alla permanenza del

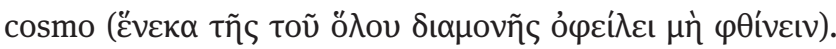

- In Lucr. 2.1120-1123; 1130 (omnibus hic aetas debet consistere rebus, / hic natura suis refrenat viribus auctum. / Nam quaecumque vides hilaro grandescere adauctu / paulatimque gradus aetatis scandere adultae [...] donec alescendi summum tetigere cacumen) la crescita dell'uomo e, parimenti, quella del mondo sono rappresentate come una salita, le cui tappe sono

53 Si noti che sono infatti riconducibili alla scuola peripatetica anche la critica all'assurdità del mito degli Sparti e la tendenza a razionalizzarlo mediante interpretazioni storico-razionalistiche di segno "palefatiano"; cfr. Ramelli-Lucchetta 2004, 226-228. Del resto, nel testo filoniano non mancano altre rielaborazioni e "falsificazioni": si pensi alla sorte dei capp. 117-149 (cfr. pp. 169178) dove, come dimostrato da Sedley, probabilmente si crea ad hoc un dibattito tra Teofrasto e Zenone, dirigendo contro gli Stoici alcuni argomenti peripatetici in origine volti contro altri Presocratici. Segni di un processo di sintesi e selezione sono presenti anche nei capitoli immediatamente successivi: basti considerare che l'autore "taglia" il pensiero di Critolao a proposito delle cause esterne di rovina del mondo (assenti nel testo).

54 Cfr. Lucr. 1.75-77 unde refert nobis victor quid possit oriri, / quid nequeat, finita potestas denique cuique / quanam sit ratione atque terminus alte haerens; 2.1087-1089 (in riferimento ai mondi) quandoquidem vitae depactus terminus alte / tam manet haec et tam nativo corpore constant, / quam genus omne quod hic generatimst rebus abundans.

55 «Ritenere che alcuni uomini siano nati dalla terra già compiuti è proprio di uomini che ignorano le leggi della natura e i suoi decreti immutabili. [...]. La natura è assolutamente immutabile, poiché domina su tutto e poiché mantiene immoti i cippi di confine infissi dal principio per la stabilità di ciò che viene conosciuto una volta per tutte». 
dipinte come gradini ${ }^{56}$ che si elevano sino a un punto apicale, momento di arresto oltre il quale non è possibile spingersi. La stessa immagine ricompare, in riferimento sia ai singoli esseri viventi sia (polemicamente) al

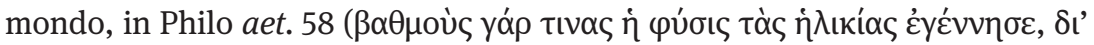

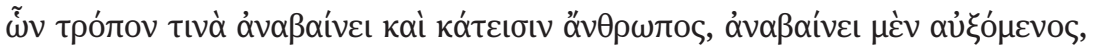

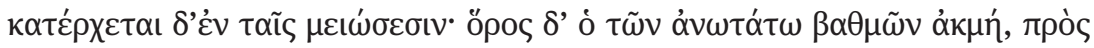

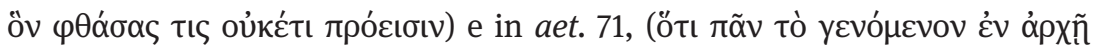

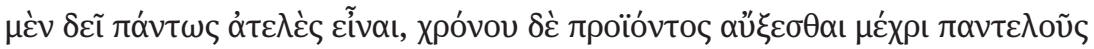

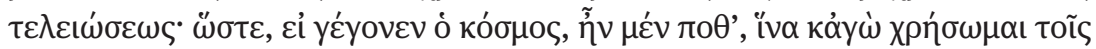

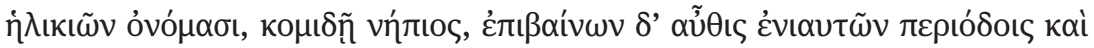

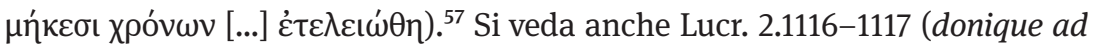
extremam crescendi perfica finem / omnia perduxit rerum natura creatrix) dove il fattore che mette in moto l'intero processo è di nuovo la natura rerum, proprio come nel passo filoniano (i் $\varphi u ́ \sigma ı)$ ).

- Il processo di "invecchiamento" del mondo è per Lucrezio lento e graduale (Lucr. 2.1123 paulatim; v. 1131 minutatim). Lo stesso concetto si ritrova in

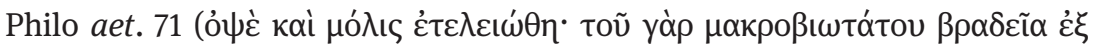

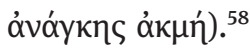

- Secondo Lucrezio, la giovinezza del mondo è contraddistinta da un saldo positivo nello scambio atomico con il mondo esterno (v. 1124 plura sibi adsumunt quam de se corpora mittunt): in sostanza, il mondo riceve più atomi di quanti ne restituisca. L'avvento della vecchiaia determina la situazione opposta: il mondo riconsegna allo spazio esterno gli atomi che aveva accumulato. L'esito di tale processo di dispersione è la dissoluzione finale (v.

56 La rappresentazione della crescita del mondo è certo presente anche in Democrito ed Epicuro, che definiscono il periodo di massimo sviluppo come $\dot{\alpha} \kappa \mu$. Tuttavia il passo riportato da Filone è il solo in cui ritroviamo anche il particolare dei $\beta \alpha \theta \mu o i ́(g r a d u s)$, stabiliti dalla natura. Si noti che l'ascendenza peripatetica di questo motivo è confermata dal fatto che, come mostra Leonardis 2018, 517-519, esso compare (con metafora biologica estesa allo sviluppo del genere umano e dei singoli popoli) anche nel logistorico varroniano Tubero de origine humana, noto-

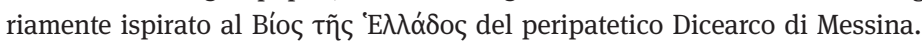

57 Philo aet. 58: «la natura infatti ha concepito alcuni gradi d'età, attraverso i quali l'uomo sale e discende: sale quando cresce, scende nella diminuzione. Il limite dei gradi diretti in alto è l'apice, raggiunto il quale uno non procede». Philo aet. 71: «ogni cosa generata deve in principio essere assolutamente incompiuta, e accrescersi con lo scorrere del tempo sino al suo totale completamento; di conseguenza, se il mondo fosse stato generato, esso sarebbe stato, per usare il lessico riferito alle età umane, soltanto un bambino, e poi, salendo nel volgere degli anni e in lunghi spazi di tempo, [...] esso sarebbe giunto a compimento». Per le molteplici rappresentazioni antiche della natura, cfr. Andreoni 1997.

58 «[l cosmo] lentamente e a fatica giunse a compimento: la strada verso l'apice dell'entità più longeva è infatti lenta». 
1132 in partem peiorem liquitur aetas). L'antitesi tra i concetti di "ricevere" e di "restituire" in relazione alla giovinezza e alla vecchiaia appare chiara-

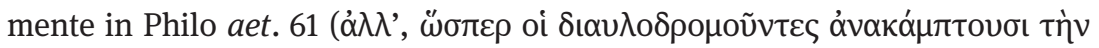

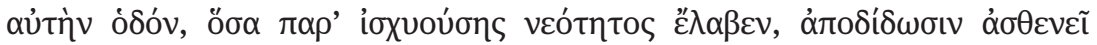
yńp $\mathbf{1}){ }^{59}$ Questo passo è inoltre degno di nota per l'immagine della lampadoforia dell'esistenza, ${ }^{60}$ che ricorre anche in Lucr. 2.78-79 (inque brevi spatio mutantur saecla animantum / et quasi cursores vitai lampada tradunt), seppur in riferimento al susseguirsi delle generazioni. Mentre per Lucrezio l'exemplum è volto a dimostrare che la morte dei singoli aggregati garantisce il permanere della materia e dunque l'eternità dell'universo (ma non dei singoli mondi), in Filone esso è usato per provare l'immortalità del genere umano a riprova dell'eternità del mondo.

- Lucrezio descrive la fine del mondo come un crollo delle mura cosmiche, “conquistate" da forze provenienti dall'esterno (Lucr. 2.1144-1145 sic igitur magni quoque circum moenia mundi / expugnata dabunt labem putrisque ruinas). Similmente, in Philo aet. 72 si chiama in causa il "perfetto muro del

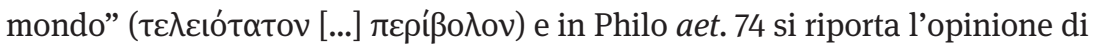
Critolao, il quale, in polemica con le dottrine stoiche, afferma che il mondo non è espugnabile ( $\dot{\alpha} \lambda \omega \tau$ tós) da nessuna delle cause che determinano la morte degli esseri viventi.

- Lucrezio dipinge la terra come una donna resa sterile "dallo spazio di tempo trascorso" (cfr. Lucr. 2.1150 iamque adeo fracta est aetas effetaque tellus; 1173-1174 omnia paulatim tabescere et ire / ad capulum spatio aetatis defessa vetusto; cfr. 5.826-827 quia finem aliquam pariendi debet habere, / destitit, ut mulier spatio defessa vetusto.). In Philo aet. 57, il medesimo argomento è

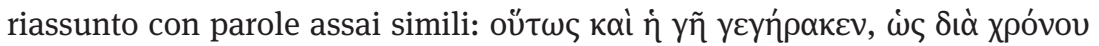
$\mu \tilde{\eta} \kappa о \varsigma ~ \varepsilon ́ \sigma \tau \varepsilon ı р \tilde{\omega} \sigma \theta \alpha \iota ~ \delta о к \varepsilon \tilde{v})$. Si noti che invece nella sezione centrale del secondo libro (vv. 589-660) Lucrezio confuta la dottrina opposta, che evidenzia la natura divina, eterna e prodigiosamente fertile della terra a vantaggio dell'umanità (cfr. e.g. vv. 594-595 nitidas fruges arbustaque laeta / gentibus humanis habet unde extollere possit). Tale dottrina è invece difesa in aet. 62-65, dove la terra è oggetto di un lungo elogio, con richiamo all'au-

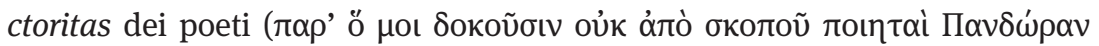

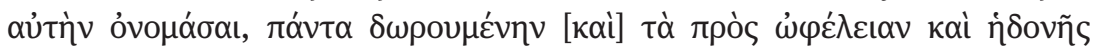

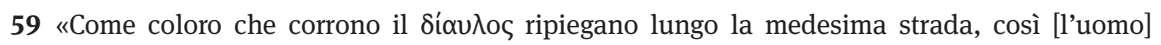
restituisce alla debole vecchiaia quanto ha ricevuto dalla forte giovinezza».

60 Per il motivo topico della lampadoforia dell'esistenza, che si ritrova già nelle Leggi di Platone (leg. 776b), cfr. Andreoni 1995, 59-60. 


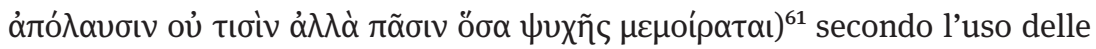
laudes terrae. ${ }^{62}$ La ripresa polemica di questi motivi da parte di Lucrezio è però sapientemente diretta non più solo contro i Peripatetici, ma, come si vedrà, anche contro l'allegorismo e il provvidenzialismo stoici. ${ }^{63}$

Significativamente, troviamo in altri passi del poema lucreziano alcune immagini che compaiono in questi capitoli filoniani, come se il poeta le avesse serbate per i propri scopi in altri punti strategici del De rerum natura:

- In 1.638-644 Lucrezio rimprovera gli stolidi ${ }^{64}$ ammiratori di Eraclito (probabilmente, di nuovo gli Stoici), ${ }^{65}$ accusati di preferire non ciò che è detto chiaramente, ma quanto è espresso sub inversis versis, considerando vere le parole che incantano l'udito e sono mascherate sotto suoni piacevoli (omnia enim stolidi magis admirantur amantque / inversis quae sub verbis latitantia cernunt, / veraque constituunt quae belle tangere possunt / auris et lepido quae sunt fucata sonore). ${ }^{66}$ Parimenti si trova in Philo aet. 56-57 una critica agli artefici di miti (forse con polemica diretta all'allegorismo stoico), accusati di colmare la vita d'inganni e d'incantare l'udito degli stolti tramite i

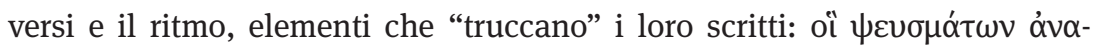

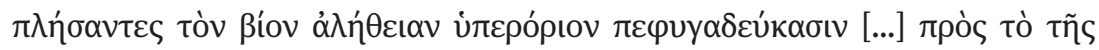

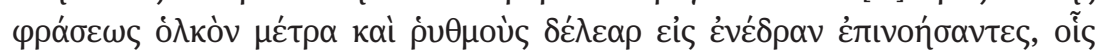

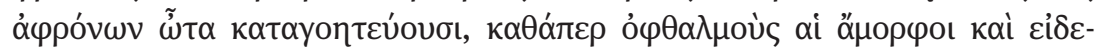

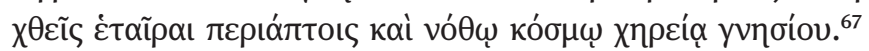

61 «Pertanto non mi pare inopportuno che i poeti l'abbiano chiamata Pandora, dato che dona ogni cosa, sia ciò che è volto all'utilità sia ciò che è volto al godimento del piacere, e non per alcuni, ma per tutti coloro che hanno avuto in sorte la vita».

62 Cfr. Landolfi 1985.

63 Cfr. pp. 162-164.

64 Il fatto che il medesimo termine sia usato soltanto in un altro contesto (1.1068), in probabile riferimento agli Stoici, accresce il sospetto che anche qui il poeta rivolga le proprie critiche proprio contro la Stoà.

65 Cfr. e.g. Craca 2000, 14-15. A proposito dei riferimenti agli Stoici in Lucr. 1.635-920, cfr. Piazzi 2005, 8-9; 26-27.

66 «Infatti gli sciocchi ammirano e amano tutto ciò che appena / distinguono celato sotto contorte parole / e affermano vero quel che può accarezzare con eleganza l'orecchio / e quel che sia camuffato in gradevole suono».

67 «Coloro che hanno colmato la vita di menzogne hanno bandito la verità oltre i confini [...] avendo escogitato, per il fascino dell'espressione, metri e ritmi come esca volta all'inganno; con tali espedienti essi incantano gli orecchi degli stolti, come le etere brutte e laide incantano gli occhi mediante ornamenti e con un trucco posticcio, in mancanza di un aspetto genuino». 
- In una parentesi poetica collocata al principio del secondo libro, Lucrezio evidenzia l'eterna giovinezza dell'universo, che si rinnova sempre (2.75-76 sic rerum summa novatur / semper) mantenendosi intatta da consunzione e vecchiaia (v. 71 incolumis videatur summa manere). In Filone (aet. 61-62) un lessico analogo è invece usato per designare l'eternità della terra e degli altri

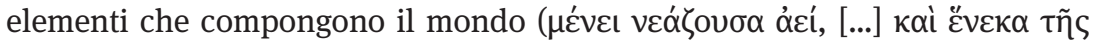

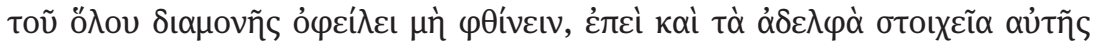

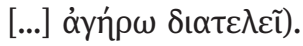

- Nel quinto libro Lucrezio riprende polemicamente la formula aristotelica che designa gli assertori della mortalità del mondo come qui ratione sua dis-

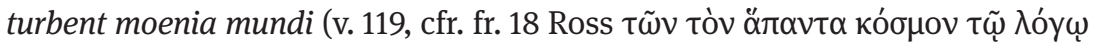
$\kappa \alpha \theta \alpha \iota p o u ́ v \tau \omega \nu) .{ }^{68}$ La formula viene richiamata anche in Filone (cfr. e. g. Philo

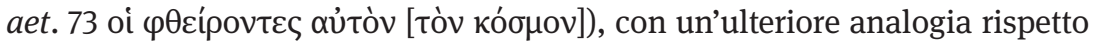
a Lucrezio, ovverosia il riferimento al crollo mura del mondo (aet. 73 tòv yà

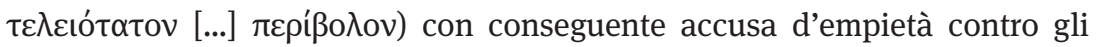

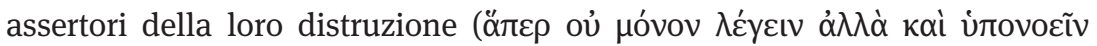

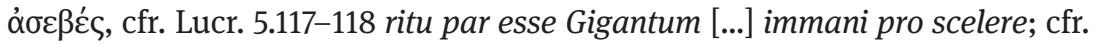
Lucr. 5.156-165).

- In Lucr. 5.228-231, per sottolineare la culpa naturae, che rende l'uomo più debole rispetto agli animali, il poeta evidenzia come i fragili neonati abbiano bisogno di madri e nutrici per essere vestiti e protetti dalle intemperie (at variae crescunt pecudes armenta feraeque / nec crepitacillis opus est nec cuiquam adhibendast / almae nutricis blanda atque infracta loquella / nec varias quaerunt vestis pro tempore caeli). ${ }^{69}$ Il medesimo motivo viene impiegato in Philo aet. 67 per negare la possibilità che la terra abbia generato e

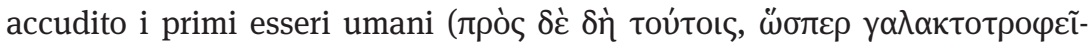

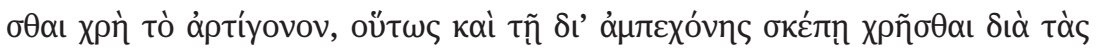

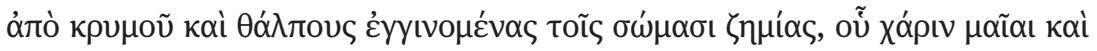

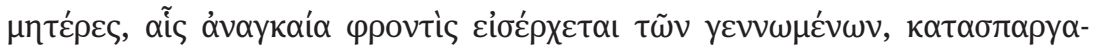

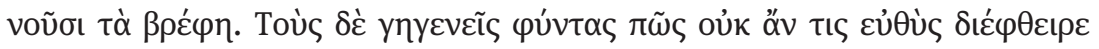

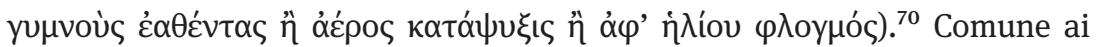
due è inoltre l'argomento della necessità delle armi per l'essere umano

68 Cfr. pp. 118-122.

69 Cfr. pp. 160-161.

70 «Oltre a ciò, come i neonati abbisognano di latte, così necessitano della protezione di un vestito, a causa dei danni provocati al corpo dal freddo e dal calore; pertanto nutrici e madri, sensibili a questa necessità dei figli, avvolgono in fasce i neonati. Di conseguenza coloro che sono generati dalla terra e lasciati nudi non dovrebbero essere distrutti da un improvviso raffreddamento dell'aria o dalla vampa solare?». 
appena generato: nel passaggio di Filone si sottolinea come la vera prerogativa dell'uomo non siano le armi, ma l'intelletto, donato dalla natura (aet. 68); Lucrezio, all'opposto, asserisce che l'uomo abbisogna delle armi poiché la natura ha donato ogni cosa a tutti gli altri esseri viventi (vv. 232234)..$^{71}$

\subsection{Alcune conclusioni}

Il notevole elenco di corrispondenze tra i versi lucreziani e le dottrine peripatetiche in Philo aet. 55-75 induce a rifiutare l'idea che il legame tra i due testi sia dovuto a una generica condivisione di motivi topici appartenenti al Bildungsgut tardo-ellenistico. Una spiegazione possibile (ma non verificabile) sarebbe allora quella di ricondurre tali analogie al comune modello del perduto De philosophia di Aristotele, confutato da Epicuro (e, di conseguenza, da Lucrezio) e invece ripreso da Critolao. Tale spiegazione è tuttavia difficilmente conciliabile con il fatto che, nel novero dei numerosi argomenti e motivi analogici del passo ripresi da Lucrezio, sono compresi quelli elaborati da Critolao e diretti contro la Stoà. ${ }^{72}$ Nella propria opera di riformulazione, Lucrezio "cancella" però gli Stoici, presentando la ratio epicurea come unico contraltare agli argomenti peripatetici. Come si vedrà, il poeta agirà in modo simile al principio della sezione escatologica del quinto libro, dipingendo i soli Epicurei come assertori della mortalità del cosmo e riducendo gli Stoici a eredi del provvidenzialismo platonico, senza alcuna menzione della dottrina cosmologica dell'ecpirosi. ${ }^{73}$ Tale operazione sistematica non impedisce a Lucrezio di recuperare alcune polemiche anti-stoiche di Critolao, reimpiegandole in altre sezioni del poema (e.g. 1.638-644; 2.589660; 5.228-231).

In alternativa alla teoria della comune dipendenza dal De philosophia, è dunque possibile formulare una diversa tesi, secondo la quale la descrizione lucreziana dello sviluppo del mondo sino alla sua distruzione in 2.1105-1174, pur prendendo le mosse dalla traccia della cosmologia epicurea, tiene in conside-

71 Naturalmente il motivo dei doni della natura agli uomini, alla base di entrambi i passi, è riconducibile al mito platonico di Epimeteo e Prometeo nel Protagora (320c-322d).

72 Come si è detto, la sezione 55-75 del trattato filoniano è infatti costruita in primis contro gli Stoici. E infatti nella sezione successiva (a partire dal cap. 76) Filone definisce gli scolarchi della cosiddetta Stoà di mezzo (Diogene di Babilonia, Boeto di Sidone, Panezio di Rodi) "vinti” dalle argomentazioni di Critolao e quindi pronti ad abbandonare il dogma veterostoico della

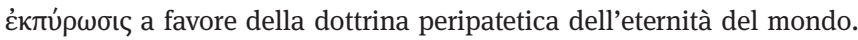

73 Cfr. pp. 249-250. 
razione la più recente difesa peripatetica dell'eternità del cosmo e della terra (riconducibile in primis a Critolao), appropriandosi dei suoi argomenti e dei suoi motivi analogici allo scopo di confutarla. La stessa presenza pervasiva del motivo analogico del mondo come essere vivente, che non ha un peso equivalente nei testi di Epicuro a noi pervenuti, è invece perfettamente motivata, se concepita in contrapposizione agli argomenti peripatetici sopra citati, che vi fanno ricorso a più riprese. Questa spiegazione si accorda al quadro delineato nei precedenti capitoli, che ha reso chiaro che Lucrezio non è un mero traduttore di Epicuro: gli argomenti di quest'ultimo non sono infatti ripresentati indistintamente, bensì oggetto di un'attenta selezione e rielaborazione in funzione di un nuovo contesto storico e, soprattutto, del pubblico latino del poema. Ulteriori conferme di questa lettura si avranno nell'analisi dei versi finali (2.1164-1174). ${ }^{74}$

Ancora una volta, come nel caso del finale del primo libro, Lucrezio non sembra sottrarsi al confronto con gli sviluppi recenti della querelle filosofica sull'eternità del mondo. È inoltre verosimile supporre che l'integrazione degli argomenti di Epicuro sia stata svolta dal poeta a partire da una fonte dossografica, probabilmente non molto dissimile, per forma e contenuti, dallo stesso De aeternitate mundi di Filone Alessandrino. ${ }^{75}$ Dunque il richiamo di Schrijvers alla trattatistica peripatetica tardo-ellenistica si rivela corretto e denso d'implicazioni per l'interpretazione globale del poema lucreziano: il poeta appare ben consapevole del contesto culturale e del dibattito filosofico della sua età, mostrandosi capace di attingere anche alla tradizione più recente. Come si detto, questo dato fondamentale non deve però condurre a ricercare, di conseguenza, tendenze più o meno scopertamente eterodosse nel De rerum natura. Potremmo quindi concludere che l'inclusione di testi poetici e filosofici successivi alla morte di Epicuro tra le fonti del finale del secondo libro non intacca, per riprendere la formula di Schiesaro, «l'ossatura epicurea» di tale passo, bensì la arricchisce e la attualizza. Le fonti peripatetiche recenziori sono interpretate da Lucrezio non solo come un obbiettivo polemico, ma anche come un repertorio d'immagini, concetti e meccanismi analogici, liberamente reimpiegati allo scopo di difendere il tracciato già definito da Epicuro.

74 Cfr. Appendice 1.

75 Non è invece possibile determinare se il poeta abbia compiuto autonomamente tale operazione o si sia invece avvalso di fonti epicuree recenziori. 


\subsection{Una pluralità di avversari: i passi anti-provvidenzialistici nel secondo libro}

Il racconto del percorso di nascita, crescita e morte del cosmo ai vv. 1105-1174 del secondo libro si contrappone quindi, implicitamente, alla dottrina dell'eternità del cosmo, associata, come vedremo anche nell'analisi del quinto libro, in primis ai Peripatetici. ${ }^{76}$ La riflessione appena svolta in relazione alla possibilità di un ricorso del poeta a fonti peripatetiche più recenti - ad esempio gli argomenti attribuiti a Critolao - apporta un'importante conferma a questa interpretazione. Nondimeno, se rivolgiamo uno sguardo all'edificio complessivo del secondo libro, notiamo che Lucrezio non contempla solo un obbiettivo polemico, bensì una pluralità di avversari. La dimostrazione finale della mortalità della terra è infatti "preparata" da una serie d'importanti passaggi confutativi, nei quali la critica è evidentemente rivolta alle dottrine del creazionismo e del provvidenzialismo, da associare in primis alle scuole platonica e stoica. ${ }^{77} \mathrm{Si}$ tratta di una considerazione rilevante, poiché il poeta si avvarrà della medesima strategia anche nella sezione escatologica del quinto libro. ${ }^{78}$

È necessario prendere le mosse dalla sezione centrale del secondo libro, dedicata anch'essa al tema della terra (vv. 589-660). Qui il poeta svolge un'archeologia del mito e del culto della terra come Magna Mater, con esplicita critica ai tratti divini attribuiti a essa dai veteres Graium docti poetae (v. 600), poi soggetti all'allegoresi dei filosofi. Al termine di questa rassegna, il poeta sottolinea come tali tratti siano lungi dalla verità (v. 645 longe sunt tamen a vera ratione repulsa), ${ }^{79}$ poiché le divinità vivono distanti dal genere umano e dal mondo, disinteressate alle loro sorti (vv. 646-651). La terra è dunque un mero aggregato atomico inanimato (v. 652 caret omni tempore sensu). ${ }^{80}$ È verosimile supporre che gli Stoici siano tra i principali obbiettivi polemici di questo passo. ${ }^{81}$

76 Cfr. pp. 164-166.

77 Per un'introduzione all'articolazione tematica di questo finale, cfr. Bonelli 1984, 131-145.

78 Cfr. pp. 162-164.

79 Si noti che anche tale espressione presenta notevoli analogie con l'espressione $\alpha \lambda \lambda \hat{\theta} \theta \varepsilon ı \alpha$

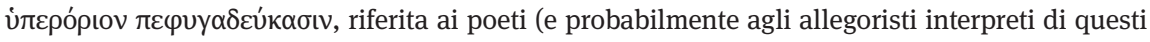
ultimi) in Philo aet. 57.

80 La "coda" dei v. 655-660 contiene, com'è noto, una concessione all'impiego del mito e dei suoi vocamina, ma alla condizione che tale uso non riconduca l'uomo ai terrori della religio: concedamus [...] / dum vera re tamen ipse / religione animum turpi contingere parcat. Su questo passo, cfr. Gale 1994, 29-33.

81 Cfr. l'ampia analisi svolta da Schmidt 1990, 113-144. La lettura anti-stoica del passo è ritenuta persuasiva anche da Sedley 1998a, 75 n. 62, Gigandet 2001, 109-121, Gigandet 2002 e Luciani 2017b. 
Una prima prova di ciò è individuabile nel fatto che la pratica dell'allegoresi, seppur comune a tutte le scuole filosofiche, era impiegata specialmente dagli Stoici e dunque a essi anzitutto associata; per giunta, proprio nelle fonti stoiche troviamo notevoli paralleli con le interpretazioni allegoriche qui criticate da Lucrezio. ${ }^{82}$ Egli nega infatti che la terra sia fonte di salvezza (salus) individuale ${ }^{83}$ e, al contempo, che sia provvista di una salus cosmica, tale da conservarla salda e stabile al centro del cosmo. Quest'ultimo aspetto è messo in rilievo sin dal principio: infatti, il primo elemento del mito della Magna Mater narrato dai dotti poeti, ossia il carro trainato da leoni, viene interpretato come simbolo della sua perfetta posizione centrale, sospesa nell'aria. ${ }^{84}$ Poco dopo, la corona muralis viene parimenti letta come simbolo della stabilità della terra, che funge da sostegno per le città del mondo in luoghi elevati. ${ }^{85}$ Queste caratteristiche rimandano chiaramente alla dottrina cosmologica che vuole la terra collocata stabilmente (e eternamente) al centro del cosmo: l'analisi del finale del primo libro ha mostrato come Lucrezio attribuisca innanzi tutto agli Stoici tale visione. ${ }^{86}$

Degni d'esame sono anche i vv. $167-182 .{ }^{87}$ Qui Lucrezio attacca infatti alcuni innominati avversari, bollati come ignari materiai, che ritengono che il mondo sia stato creato per l'umanità (hominum causa) e dunque attribuiscono ogni fenomeno a una benevola provvidenza divina: le stagioni, i raccolti (dovuti alla fertilità dei suoli), la struttura fisica dell'essere umano, che rende possibile,

82 Si vedano i vv. 644-645, dove le verità allegoriche sono dette longe a vera ratione repulsa sebbene esposte in maniera accattivante (quae bene et eximie quamuis disposta ferantur). Come si è visto, una simile critica era rivolta nel primo libro agli ammiratori di Eraclito (1.638-644), da identificare, con ogni probabilità, con gli Stoici stessi. Cfr. Gale 1994, 32-33; Craca 2000, 147-149, Piazzi 2011, 175-177 (che sottolinea le analogie tra Lucrezio e alcuni passi filodemei anti-stoici) e Montarese 2012, 199-208, che mostra invece dubbi sul fatto che Lucrezio si stia riferendo specificamente agli Stoici. A proposito dell'attacco all'allegorismo e al provvidenzialismo stoici nel secondo libro, culminante nell'allusione polemica al mito dell'aurea funis in 2.1153-1155, cfr. Schmidt 1990, 144-151 e Galzerano 2020.

83 Il riferimento al culto di Tacita Muta rappresenta un'allusione ai misteri di salvazione associati a Cibele. Cfr. Craca 2000, 73-74.

84 Cfr. 2.601-604: sedibus in curru biiugos agitare leones, / aeris in spatio magnam pendere docentes / tellurem neque posse in terra sistere terram. Cfr. Schmidt 1990, 126-127.

85 Cfr. 2.606-607: muralique caput summum cinxere corona, / eximiis munita locis quia sustinet urbis. Si veda l'analisi di Schmidt 1990, 131, che richiama in particolare il parallelo offerto dall'allegorista stoico Cornuto, theol. Graec. 6 (S. 6.3-6 Lang), il quale ipotizza che la corona muralis sia infatti da interpretare come simbolo del mondo in quanto cosmopoli.

86 Cfr. pp. 49-67.

87 Secondo Sedley 2011, 159-161, l'obbiettivo polemico del passo è Platone. Per un'interpretazione anti-stoica dei vv. 167-182 cfr. Schmidt 1990, 152-160. 
tramite la riproduzione, la sopravvivenza della specie. Contro costoro, il poeta rimarca l'imperfezione del mondo (culpa), prova irrefutabile dell'inesistenza di un progetto divino per l'umanità (vv. 180-181 nequaquam nobis divinitus esse creatam / naturam mundi). Questi argomenti saranno ripresi nel quinto libro e dunque saranno esaminati più esaustivamente più avanti: ${ }^{88}$ tuttavia, importa qui evidenziare che i nemici contemplati in questo passo non possono essere $\mathrm{i}$ Peripatetici, che ritenevano il mondo increato. Platonismo e Stoicismo sono dunque i soli possibili avversari contro i quali Lucrezio dirige la propria confutazione. ${ }^{89}$

Per quanto concerne la parentesi anti-provvidenzialistica che precede la sezione finale del libro (vv. 1090-1104), è innanzi tutto importante ricordare che questi versi seguono la dimostrazione dell'esistenza d'infiniti mondi e generi umani (vv. 1048-1089). L'accettazione di tale principio rende ancor più assurda e inverosimile la concezione di una divinità ubiqua e benevola nei confronti del genere umano, ma soprattutto capace di amministrare, nello stesso momento, ciascuno dei mondi nell'immensità dell'universo. ${ }^{90}$ Nei versi finali, la polemica accenna di nuovo al motivo della culpa naturae e, in particolare, al caos che regna nella società umana, dove spesso gli innocenti e non i colpevoli sono colpiti dalle disgrazie. Come si vedrà, anche questo tema sarà ripreso nel corso del quinto libro, in un contesto polemico che pare diretto in primis contro il provvidenzialismo stoico. ${ }^{91}$

La rassegna appena presentata dimostra che il poeta ha concepito l'architettura del secondo libro in maniera analoga a quella della sezione escatologica del quinto: anche qui infatti la vera e propria confutazione degli argomenti peripatetici in difesa dell'eternità del mondo (vv. 235-415) è preceduta da un

88 Cfr. pp. 148-156.

89 A proposito degli obbiettivi polemici di questo passo, cfr. Fowler 2002, 235. Una buona testimonianza del fatto che le tesi attaccate non siano soltanto quelle stoiche si ritrova nelle argomentazioni provvidenzialistiche svolte dal Socrate dei Memorabilia di Senofonte (mem. 1.4.6-17): qui infatti il filosofo svolge un notevole elenco di doni divini, che dimostrano la benevolenza degli dèi verso l'umanità; tali doni includono sia fattori ambientali (come le stagioni) sia le caratteristiche uniche dell'esistenza e del corpo dell'uomo. Si veda in proposito Sedley 2011, 93-96.

90 Un ottimo termine di paragone per comprendere la visione del mondo osteggiata da Lucrezio è rappresentato, a mio parere, dai versi iniziali dei Fenomeni di Arato: qui troviamo infatti l'idea di un dio che pervade il mondo intero (vv. 2-4), e che governa i corpi celesti in favore dell'umanità (vv. 10-14).

91 Cfr. pp. 000. Per la rilevanza di questa tematica per gli Stoici, basti qui ricordare l'incipit del De providentia di Seneca (dial. 1.1): quaesisti a me, Lucili, quid ita, si providentia mundus ageretur, multa bonis viris mala acciderent. 
attacco al creazionismo e alla dottrina del cosmo creato hominum causa (vv. 91234). 